\title{
Comparing total medical expenditure between patients receiving direct oral anticoagulants vs warfarin for the treatment of atrial fibrillation: evidence from VA-Medicare dual enrollees
}

\author{
Edwin S Wong, PhD; Nicolae Done, PhD; Molly Zhao, BA; Adam B Woolley, PharmD; Julia C Prentice, PhD; \\ and Hillary J Mull, PhD
}

\section{What is already known about this subject}

- Newer direct oral anticoagulants (DOACs) are an alternative to warfarin for the treatment of atrial fibrillation.

- DOACs have been shown to be either noninferior or superior to warfarin on a variety of outcomes in clinical trials.
What this study adds

- Knowledge is incomplete about the generalizability of previous findings from trial settings to real-world patient populations that differ in factors such as socioeconomic status and comorbidity.

- This study presents new evidence comparing total medical expenditure among Veterans Affairs Health Care System patients receiving warfarin and DOACs.

\author{
Author affiliations \\ Edwin S Wong, PhD, VA Puget Sound Health \\ Care System, Seattle, WA, and Department \\ of Health Services, University of Washington, \\ Seattle. Nicolae Done, PhD, and Molly Zhao, \\ BA, Center for Healthcare Organization \\ and Implementation Research (CHOIR), \\ VA Boston Healthcare System, Boston, MA. \\ Julia C Prentice, PhD, Center for Healthcare \\ Organization and Implementation \\ Research (CHOIR), VA Boston Healthcare \\ System, Boston, MA, and Department of \\ Psychiatry, Boston University, Boston, MA. \\ Adam B Woolley, PharmD, Northeastern \\ University, Boston, MA. Hillary J Mull, PhD, \\ Center for Healthcare Organization and \\ Implementation Research (CHOIR), VA \\ Boston Healthcare System, Boston, MA, and \\ Department of Surgery, Boston University, \\ Boston, MA. \\ AUTHOR CORRESPONDENCE: \\ Edwin S Wong, 206.277.4703; \\ eswong@uw.edu
}

J Manag Care Spec Pharm. 2021;27(8):1056-66

Copyright $\odot 2021$, Academy of Managed Care Pharmacy. All rights reserved. 


\section{ABSTRACT}

BACKGROUND: Direct oral anticoagulants (DOACs) are an alternative to warfarin for treatment of atrial fibrillation (AF). Evidence demonstrating the efficacy and safety of DOACs has primarily been from clinical trial settings. The real-world effectiveness of DOACs in specific nontrial populations that differ in age, comorbidity burden, and socioeconomic status is unclear.

OBJECTIVE: To compare total downstream medical expenditure between AF patients treated with warfarin and DOACs dually enrolled in the Veterans Affairs (VA) Healthcare System and fee-forservice Medicare.

METHODS: This was an exploratory treatment effectiveness study that analyzed VA administrative data and Medicare claims. We examined patients with an incident diagnosis for $\mathrm{AF}$ and initiated warfarin or DOAC treatment between 2012 and 2015. The primary outcome was total medical expenditure over 3 years following treatment initiation. To address potential informative censoring, we applied a multipart estimator that extends traditional 2-part models to separate differences between groups due to survival and cost accumulation effects. Inverse probability weighting was applied to address potential treatment selection bias.

RESULTS: We identified 31,276 and 17,021 patients receiving warfarin and DOACs, respectively. Mean unadjusted (SD) expenditure was higher for warfarin $(\$ 56,265[\$ 96,666])$ compared with DOAC patients $(\$ 32,736[\$ 52,470])$. Compared with patients receiving DOACs, adjusted 3-year expenditure was $\$ 25,688(P<0.001)$ higher for patients receiving warfarin.

CONCLUSIONS: VA patients with AF initiating warfarin incurred markedly higher downstream expenditure compared with similar patients receiving DOACs. The benefits of DOACs found in previous clinical trials were present in this population, suggesting that these DOACs may be the preferred option for treatment of AF in older VA patients.

Atrial fibrillation (AF) is the second most common cardiovascular condition that affects up to 6.1 million individuals in the United States. ${ }^{1}$ One aspect of AF treatment focuses on preventing blood clots to reduce stroke risk. Traditionally, warfarin, a vitamin-K antagonist, has been used to reduce stroke risk. However, warfarin requires regular laboratory monitoring to ensure safety and efficacy, is associated with several adverse effects and drug/food interaction risks, and has low convenience ratings by patients. ${ }^{2}$

Newer direct oral anticoagulants (DOACs) are an alternative option that does not require laboratory monitoring because of their more predictable pharmacokinetics. DOACs have been shown to be either noninferior or superior to warfarin on a variety of outcomes in clinical trials., ${ }^{3,4}$ In addition, DOACs may be cost-effective for certain patient populations. ${ }^{5}$ Much of the evidence demonstrating benefits of DOACs over warfarin has been in clinical trial settings, which may have limited generalizability in real-world practice. Studies comparing DOACs with warfarin in real-world settings have produced mixed results. ${ }^{6}$

The generalizability of findings from clinical trials to patients enrolled in the Veterans Affairs (VA) Healthcare System is unclear. VA patients have complex medication regimens and often obtain medications from VA and nonVA sources. ${ }^{7}$ Also, VA patients have greater socioeconomic challenges, a higher rate of substance abuse, and greater risk of cognitive impairment compared with the general population. ${ }^{7-9}$ These factors may reduce patients' ability to remain adherent to anticoagulation therapy. This is particularly important for DOACs, which have a shorter duration of blood thinning effect than warfarin, so missed doses leave a patient unprotected against stroke. Previous trials excluded individuals deemed unreliable or who had conditions compromising their ability to take DOACs safely, such as alcohol or drug abuse. Also, storage of DOACs such as dabigatran have stricter requirements, including the need for tight humidity control.

In addition, the VA population is older and has a high burden of illness, which may compromise the efficacy of DOACs compared with warfarin. ${ }^{10}$ A subanalysis of RE-LY, the pivotal dabigatran trial, found a trend toward more frequent major bleeding for patients aged 75 years and older on dabigatran compared with warfarin. ${ }^{11}$ Although recent studies have demonstrated the safety of apixaban in AF patients with renal impairment, ${ }^{12}$ a common condition in the VA population, warfarin has traditionally been the preferred anticoagulant for these patients..$^{13}$ Unlike warfarin, there is no reliable approach to measuring blood levels of dabigatran; therefore, its impact on coagulation assays may not correlate to clinical outcomes. ${ }^{14}$

Previous studies have focused primarily on mortality and clinical outcomes such as stroke and bleeding events. ${ }^{15-21}$ Limited evidence on the downstream medical expenditure following initiation of pharmacotherapy for AF currently exists. Two studies compared total and stroke-/systemic embolism (SE)-related costs between AF patients treated with warfarin and those treated with apixaban, dabigatran, or riveroxaban. ${ }^{22,23}$ Similar to existing research on clinical outcomes, these studies examined non-VA populations, so generalizability to VA enrollees with $\mathrm{AF}$ is unknown. Furthermore, a limitation of previous economic studies is differences in rates of death between patients receiving warfarin and DOACs was not accounted for. 
All else being equal, if DOACs are at least comparable with warfarin in terms of reducing stroke and minimizing risk of adverse events such as bleeding, then downstream medical expenditure should be lower for patients prescribed DOACs who do not require regular laboratory monitoring. However, if the efficacy of DOACs compared with warfarin is different in the VA population compared with trial populations or adherence is suboptimal, then patients prescribed DOACs may instead incur higher downstream medical expenditure because of factors such as greater adverse events.

This study examined whether advantages of DOACs found in previous trials translated to the VA population by comparing total downstream medical expenditure over 3 years between AF patients treated with warfarin and DOACs. To address potential biases from differential attrition stemming from death, we applied a unique multipart estimator that separated the expenditure differences between warfarin and DOAC patients due to respective survival and expenditure intensity effects. Our approach comprehensively captured patients' future expected medical resource use that is relevant for integrated health systems such as the VA, which is responsible for comprehensively managing care for its patient population.

\section{Methods}

\section{STUDY DESIGN AND DATA SOURCES}

This retrospective national cohort study used data from several sources. The primary source was administrative data from the VA's Corporate Data Warehouse, which contains extensive information tracking of all care delivered at VA facilities nationally. This includes data on patient demographics, diagnosis and procedure codes, health service utilization, and prescription drug fills. VA administrative data were linked to 2 sources of expenditure data. The first source was data from the VA Health Economics Resource Center, which contain medical encounter-level cost estimates constructed to be proportional to Medicare reimbursement rates for similar services. ${ }^{24}$ The second source was the Managerial Cost Accounting System pharmacy extracts, which includes information on drug and dispensing costs. ${ }^{25}$

VA data were linked to (1) Medicare claims, which include information on care received by patients through traditional fee-for-service Medicare and (2) the Medicare Part D Event File, which contains information on the cost of drugs reimbursed by Medicare. Medicare data were included to construct more complete measures of expenditure and to ensure completeness in the construction of baseline characteristics and acute events such as stroke and acute myocardial infarction. Data from respective VA and Medicare sources were linked using unique patient identifiers.

Data from the VA External Peer Review Program were used to ascertain measures of primary care clinical quality. ${ }^{26}$ These data were collected through chart abstraction of a random sample of patients at each VA facility. Quality data were aggregated to the facility level and linked to patient-level data through unique VA facility identifiers that capture where patients receive primary care.

\section{STUDY SAMPLE}

We identified 282,163 patients who received care from the $\mathrm{VA}$ and had at least 1 prescription fill for warfarin or a DOAC during the period between January 1, 2012, and December 31, 2015 (Figure 1). We defined the date of the first observed drug fill as the index date. We excluded 127,988 patients who did not have a diagnosis of nonvalvular AF (International Classification of Diseases, Ninth/Tenth Revision, Clinical Modification [ICD-9-CM] codes 427.3, 427.31 and 427.32 before October 1, 2015, and ICD-10-CM code I48.x thereafter) in the 12 months before the index date..$^{27}$

Next, we excluded patients missing facility-level identifiers $(n=604)$. To ensure that we had complete data on VA patients dually enrolled in fee-for-service Medicare, we excluded patients aged under 65 years on the index date or who were enrolled in a Medicare Advantage (MA) plan at any time between the index date and the end of the inclusion period (December 31, 2015; $\mathrm{n}=56,362$ ), since individual claims are not available through MA. We then excluded patients with an index drug fill covered by Medicare $(n=18,466)$ to ensure that the study sample consisted of patients that were more likely to have coagulation monitoring interactions through the VA.

Subsequently, we dropped 27,249 patients who switched or discontinued anticoagulants within 12 months after the index date. Patients who switched or discontinued anticoagulants before death and had less than a full year of expenditure did not contribute information to empirical models (see the Statistical Analysis subsection for more detail).

Finally, we excluded 3,197 patients with missing covariate data. The final study sample included 48,297 patients.

\section{OUTCOMES}

The primary outcome was total medical expenditure following initiation of anticoagulation treatment for AF over a period of up to 3 years. This included expenditures for utilization of inpatient services and outpatient services delivered within VA facilities. Inpatient care included acute and nonacute (eg, long-term care) services. VA expenditures 


\section{FIGURE 1 Sample Study Selection}

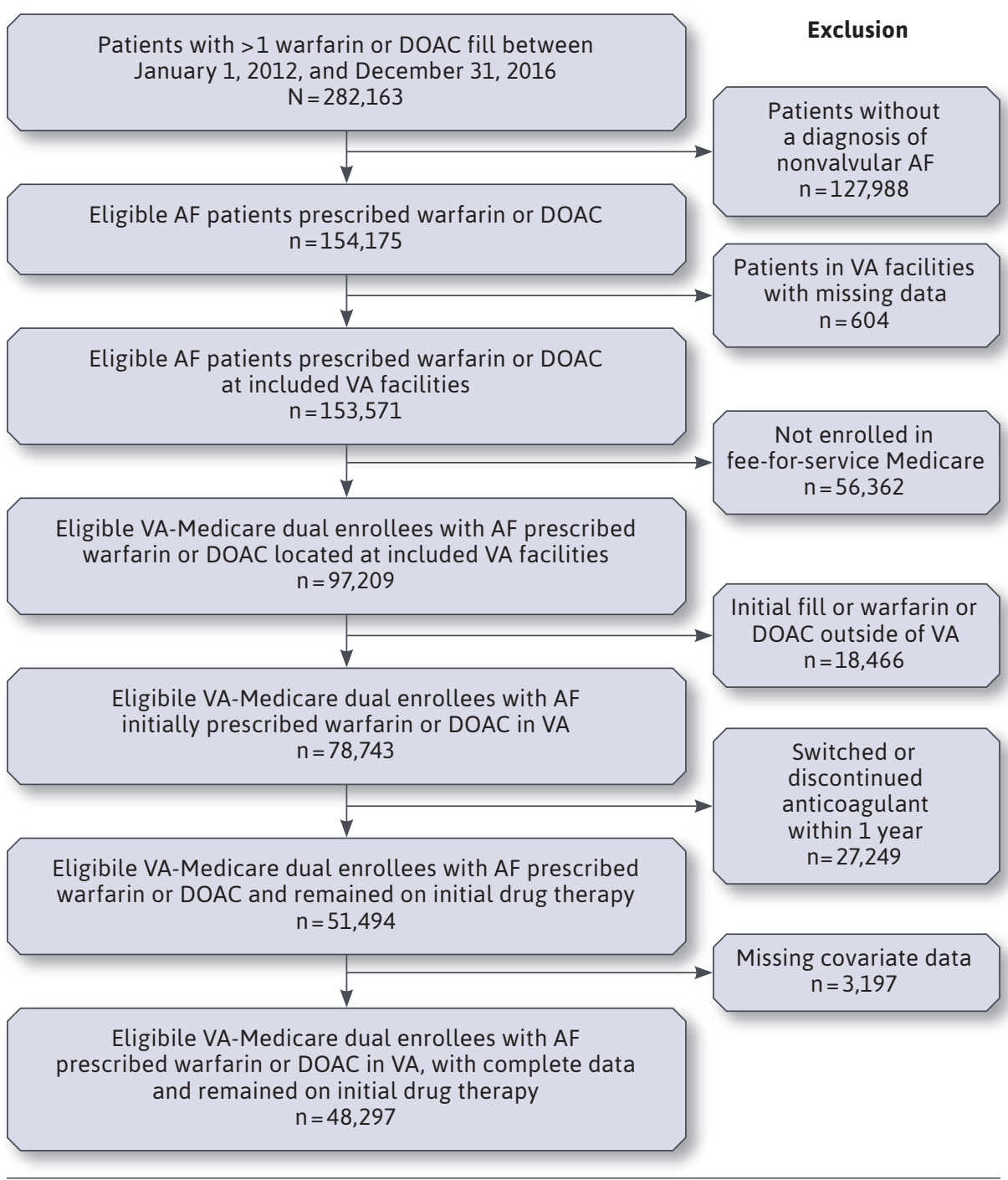

$A F=$ atrial fibrillation; $D O A C=$ direct oral anticoagulant; $V A=$ Veterans Affairs.

also included the costs of care contracted to non-VA providers. VA medical expenditures were summed with expenditures for medical services reimbursed by Medicare, which included inpatient, outpatient, and nursing home services. Patient-level medical expenditure was calculated from the date of initiation of anticoagulation until the earliest of the following: (1) date of death, (2) date of described. All expenditures were calculated from the perspective of the public payer, reflecting the cost of medical services and paid for by the VA and Medicare. We adjusted expenditures for inflation using the Consumer Price Index (CPI), reflecting 2018 constant dollars. ${ }^{28}$ Previous research has demonstrated that the general CPI exhibits better performance in adjusting for inflation compared with its medical component. ${ }^{29}$

To facilitate analysis that accounts for mortality over the 3-year follow-up period, we partitioned total expenditure into 3 variables that captured expenditure in each of 3 follow-up years. These annual measures were accompanied by a count variable denoting years of survival. Data on survival were available for up to 5 years.

\section{COVARIATES}

We ascertained an extensive set of variables related to outcomes among patients treated with oral anticoagulants, measured using VA administrative data linked with VA facility-level survey data and Medicare claims. These data included variables measured at baseline capturing patient demographics, stroke risk, fall risk, bleeding risk, history of hemorrhage, comorbidity, use of prescription medications, and clinical quality of patients' usual care facilities.

Stroke risk measures included the $\mathrm{CHA}_{2} \mathrm{DS}_{2}$-VASc (congestive heart failure, hypertension, age $\geq 75$, diabetes mellitus, prior transient ischemic attack or stroke, vascular disease, age 65-74, sex category) score, which is used by the American College of Cardiology and the American Heart Association to determine anticoagulation therapy recommendations. The $\mathrm{CHA}_{2} \mathrm{DS}_{2}$-VASc is considered a gold standard for estimating stroke risk. ${ }^{30}$

Measures of bleeding risk included the validated ATRIA, HAS-BLED, 


\begin{tabular}{l|c|c}
\hline \multicolumn{1}{c|}{ TABLE 1 } & \multicolumn{2}{c}{$\begin{array}{l}\text { Frequency of } \\
\text { Patients by } \\
\text { Prescribed } \\
\text { Anticoagulant }\end{array}$} \\
\hline Index drug & $\mathbf{n}$ & $\%$ \\
\hline Apixaban & 7,579 & 15.69 \\
\hline Dabigatran & 4,985 & 10.32 \\
\hline Edoxaban & 12 & 0.02 \\
\hline Rivaroxaban & 4,445 & 9.20 \\
\hline Warfarin & 31,276 & 64.76 \\
\hline Total & $\mathbf{4 8 , 2 9 7}$ & $\mathbf{1 0 0 . 0 0}$ \\
\hline
\end{tabular}

and HEMORR ${ }_{2}$ HAGES measures. ${ }^{31-33}$ Construction of HAS-BLED omitted labile international normalized ratio (INR) values due to the lack of available data for newly diagnosed AF patients. This modified HAS-BLED score has been shown to identify patients at high bleeding risk despite lacking INR values..$^{34}$ As in previous research, construction of $\mathrm{HEMORR}_{2} \mathrm{HAGES}$ excluded genetic factors, which were unavailable in administrative data. ${ }^{35}$

Demographics included age, sex, and race/ethnicity. Comorbidities included 30 indicator variables denoting the presences of conditions within the validated Agency for Healthcare Research and Quality Comorbidity Software. Prescription medications included 20 indicator variables denoting whether patients were prescribed cardiac-related and other medications at baseline.

Clinical quality included variables denoting the percentage of a facility's eligible patients meeting clinical practice guidelines (eg, percentage of patients with blood pressure $<140 / 90$ $\mathrm{mm} \mathrm{Hg}$ ). We added quality indicators to proxy for facility-level prescribing practices that potentially influenced whether patients were prescribed warfarin or DOACs.

\section{PROPENSITY SCORE WEIGHTS}

We applied inverse probability of treatment weighting (IPTW) to address potential nonrandom assignment of anticoagulation therapy. Weights were constructed as the inverse of propensity scores estimated using generalized boosted machine (GBM), a machine learning approach that models the probability of initiating treatment with warfarin (compared with DOACs) using an ensemble of regression trees. The key advantage of GBM is facilitation of the inclusion of nonlinear transitions and interactions of covariates into the propensity score model to achieve greater covariate balance ${ }^{36}$ Predictors in the propensity score model included patient characteristics and facility-level measures of quality presented in the previous subsection.

\section{STATISTICAL ANALYSIS}

For statistical analysis, we first described the proportion of patients surviving to each year using the Kaplan-Meier estimator. Next, we presented univariate statistics of expenditure in each year and in total across the 3 years. The primary analysis separately examined medical and anticoagulant expenditure using a multipart estimator developed by Basu and Manning (2010). This approach estimates differences in total 3-year medical or anticoagulant expenditure between AF patients prescribed warfarin and DOACs. ${ }^{37}$ This estimator accounts for differences in expenditures that arise from censoring due to death or end of study follow-up. In the first part, we used a parametric survival model to estimate the survival and hazard functions denoted $\mathrm{S}_{\mathrm{j}}(\mathrm{X})$ and $\mathrm{h}_{\mathrm{j}}(\mathrm{X})$. The second part modeled expected expenditures in a given year among patient-year observations where death was observed, denoted $\mu_{1 j}(X)$, using a generalized linear model (GLM). Finally, the third part required modeling expected expenditures in a given year among patient-year observations where a full year of expenditure was observed, denoted $\mu_{2 \mathrm{j}}(\mathrm{X})$, also using a GLM.

Using the method of recycled predictions, we calculated expected expenditure over the 3-year period as the sum of expenditure in each of 3 individual years, weighted by the probability of survival in each year. In each year, expected expenditure was calculated as the weighted average of adjusted expenditure conditional on (1) surviving the full year $\left(\mu_{2 j}[X]\right)$, and (2) dying within the year $\left(\mu_{1 j}[X]\right)$, with adjusted hazard rates serving as weights. Death dates are stochastic over the course of the year, so adjusted expenditure $\mu_{1 j}(X)$ was calculated as the weighted sum of recycled predictions conditional on the proportion of the year survived, with empirical distribution of death times within the year serving as weights. Note that expected expenditure was calculated for all patient-year observations. For patients not alive for a given patient-year observation, expected expenditure was calculated out-of-sample.

We defined the treatment effect as the difference in expected 3-year expenditure attributable to treatment with warfarin relative to DOACs. We calculated this difference for each patient, which is a function of $\hat{\mu}_{1 j}(X)$, $\hat{\mu}_{2 \mathrm{j}}(\mathrm{X}), \hat{\mathrm{S}}_{\mathrm{j}}(\mathrm{X}), \mathrm{H}_{\mathrm{j}}(\mathrm{X})$ and the difference in each of these quantities conditional on receiving warfarin and DOACs, respectively. Each of these quantities was calculated using the method of recycled predictions. Patient-level differences were then averaged to generate an average treatment effect (Supplementary File 1, available in online article). 
TABLE 2 Descriptive Statistics Between Patients Prescribed Warfarin and DOACs

\begin{tabular}{|c|c|c|c|c|c|c|c|}
\hline & $\begin{array}{c}\text { Warfarin } \\
\mathrm{n}=31,276\end{array}$ & $\begin{array}{c}\text { DOAC } \\
n=17,021\end{array}$ & SMD $^{\mathbf{a}}$ & & $\begin{array}{l}\text { Warfarin } \\
\mathrm{n}=\mathbf{3 1}, \mathbf{2 7 6}\end{array}$ & $\begin{array}{c}\text { DOAC } \\
n=17,021\end{array}$ & SMD $^{\mathbf{a}}$ \\
\hline \multicolumn{4}{|l|}{ Demographics } & \multicolumn{4}{|l|}{ Mental health comorbidities, $\%$} \\
\hline Age, mean (SD) & $74.7(7.8)$ & $74.8(7.8)$ & -0.008 & PTSD & 10.8 & 10.9 & -0.004 \\
\hline Female, \% & 1.3 & 1.4 & -0.006 & Substance abuse & 2.4 & 2.3 & 0.010 \\
\hline Race: Black, \% & 7.4 & 6.5 & 0.036 & \multicolumn{4}{|l|}{ Physical comorbidities, \% } \\
\hline Race: Other, \% & 2.9 & 2.7 & 0.012 & Chronic pulmonary disease & 41.0 & 39.9 & 0.021 \\
\hline \multicolumn{4}{|l|}{ Stroke risk, mean (SD) } & Diabetes & 46.8 & 45.0 & 0.036 \\
\hline $\mathrm{CHA}_{2} \mathrm{DS}_{2}$-VASc score & $3.9(1.5)$ & $3.8(1.5)$ & 0.016 & Fluid/electrolyte disorders & 28.6 & 27.3 & 0.029 \\
\hline \multicolumn{4}{|l|}{ Bleeding risk, mean (SD) } & Pulmonary circulatory disorders & 11.6 & 11.0 & 0.021 \\
\hline ATRIA & $3.4(2.4)$ & $3.3(2.5)$ & 0.037 & Renal disease & 21.8 & 20.2 & 0.039 \\
\hline HAS-BLED & $2.4(0.9)$ & $2.4(0.9)$ & 0.011 & \multicolumn{4}{|l|}{ Antihypertensives, \% } \\
\hline $\mathrm{HEMORR}_{2} \mathrm{HAGES}$ & $2.7(1.7)$ & $2.8(1.7)$ & 0.028 & (1) & 45.1 & 43.7 & 0.028 \\
\hline \multicolumn{4}{|c|}{ Cardiovascular comorbidities, $\%$} & Beta blockers & 72.7 & 70.8 & 0.043 \\
\hline Acute myocardial infarction & 18.5 & 17.5 & 0.024 & Calcium channel blockers & 41.2 & 40.0 & 0.024 \\
\hline Congestive heart failure & $\begin{array}{l}40.0 \\
907\end{array}$ & 39.5 & 0.010 & Diuretics & 48.1 & 45.6 & 0.050 \\
\hline $\begin{array}{l}\text { Hypertension } \\
\text { Peripheral artery disease }\end{array}$ & 24.4 & 24.2 & 0.005 & Other antihypertensive & 12.7 & 11.7 & 0.031 \\
\hline retiplier al al tery uisedse & 13.4 & 13.4 & -0.001 & \multicolumn{4}{|l|}{ Other cardiovascular medications, \% } \\
\hline Valvular heart disease & 26.7 & 26.6 & 0.001 & Antiarrhythmics & 11.6 & 12.0 & -0.010 \\
\hline Venous thromboembolism & 8.0 & 7.4 & 0.024 & Angiotensin receptor inhibitors & 14.9 & 14.8 & 0.001 \\
\hline \multicolumn{4}{|l|}{ Hemorrhage, \% } & Antilipemics & 71.6 & 71.4 & 0.004 \\
\hline Any hemorrhage & 13.3 & 12.9 & 0.011 & Antiplatelets & 14.9 & 14.8 & 0.003 \\
\hline \multicolumn{4}{|l|}{ Markers of fall risk, \% } & \multicolumn{4}{|l|}{ Other prescription medications, \% } \\
\hline Excessive fall risk & 21.4 & 21.2 & 0.005 & Antianginals & 19.3 & 17.9 & 0.036 \\
\hline Neurological disorders & 10.0 & 10.2 & -0.005 & Hypoglycemics & 27.7 & 26.4 & 0.029 \\
\hline \multicolumn{4}{|c|}{ Mental health comorbidities, \% } & Insulins & 16.6 & 15.2 & 0.039 \\
\hline Dementia & 7.2 & 7.7 & -0.017 & Nonopioid analgesics & 36.2 & 33.9 & 0.049 \\
\hline Depression & 18.5 & 18.2 & 0.009 & Opioid analgesics & 35.2 & 33.2 & 0.043 \\
\hline
\end{tabular}

${ }^{a}$ Estimates reflect differences before rounding.

ACEI = angiotensin-converting enzyme inhibitor; $C H A 2 D S 2-V A S C=$ congestive heart failure, hypertension, age $\geq 75$, diabetes mellitus, prior transient ischemic attack or stroke, vascular disease, age 65-74, sex category; PTSD=posttraumatic stress disorder; SMD=standardized mean difference; TIA =transient ischemic attack.

Standard errors for treatment effect estimates were calculated using the Krinsky and Robb procedure. All statistical analyses were performed using Stata version 15.0 (StataCorp) and RStudio version 0.99.891 (RStudio Inc.). A nominal $\mathrm{P}$ value of 0.05 was used to assess all statistical hypotheses. This study was approved by the institutional review boards at the VA Boston Healthcare System and the VA Puget Sound Healthcare System.

\section{Results}

\section{DESCRIPTIVE STATISTICS}

Table 1 presents a tabulation of patients by DOAC. A total of 31,276 and 17,021 patients received warfarin and DOACs, respectively. Table 2 compares selected characteristics of patients receiving warfarin and DOACs. Standardized mean differences (SMDs) between groups were less than 0.1 for all variables after applying IPTW, a common threshold for 


\section{TABLE 3 Unadjusted Medical Expenditures by Follow-Up Year}

\begin{tabular}{|c|c|c|c|c|c|c|}
\hline & Mean & SD & $\begin{array}{c}\text { 25th } \\
\text { percentile }\end{array}$ & Median & $\begin{array}{c}\text { 75th } \\
\text { percentile }\end{array}$ & $\mathbf{N}$ \\
\hline \multicolumn{7}{|l|}{ All } \\
\hline Total 3 years & 45,218 & 79,922 & 6,805 & 21,739 & 57,340 & 48,297 \\
\hline Year 1 & 32,423 & 65,582 & 4,532 & 13,830 & 38,925 & 48,297 \\
\hline Year 2 & 26,216 & 47,126 & 4,164 & 10,055 & 27,773 & 15,047 \\
\hline Year 3 & 27,149 & 55,042 & 4,073 & 9,947 & 27,991 & 6,803 \\
\hline \multicolumn{7}{|l|}{ Warfarin } \\
\hline Total 3 years & 56,265 & 96,666 & 10,864 & 30,361 & 72,052 & 31,276 \\
\hline Year 1 & 39,951 & 80,606 & 6,848 & 18,819 & 50,500 & 31,276 \\
\hline Year 2 & 28,568 & 50,412 & 4,484 & 11,095 & 30,507 & 12,686 \\
\hline Year 3 & 28,196 & 58,372 & 4,166 & 10,751 & 30,085 & 6,174 \\
\hline \multicolumn{7}{|l|}{ DOACs } \\
\hline Total 3 years & 32,736 & 52,470 & 4,034 & 14,558 & 40,504 & 17,021 \\
\hline Year 1 & 23,916 & 41,026 & 2,975 & 9,289 & 27,726 & 17,021 \\
\hline Year 2 & 22,060 & 40,352 & 3,587 & 8,154 & 23,102 & 2,361 \\
\hline Year 3 & 24,601 & 45,863 & 3,845 & 8,411 & 22,745 & 629 \\
\hline
\end{tabular}

${ }^{a}$ All estimates are inflation adjusted to represent 2018 constant dollars.

$D O A C=$ direct oral anticoagulant.

determining covariate balance. ${ }^{38}$ Supplementary Figure 1 (available in online article) presents SMDs for all covariates before and after IPTW.

On average, patients in both groups were aged nearly 75 years and predominately male. Patients had an average $\mathrm{CHA}_{2} \mathrm{DS}_{2}$-VASc of nearly 4, indicating high stroke risk. Cardiovascular comorbidities were common in both groups, including over $40 \%$ with congestive heart failure and $24 \%$ with peripheral artery disease. Nearly $11 \%$ of patients had a diagnosis of posttraumatic stress disorder, which is more common in a VA population. Before IPTW, the most substantial difference between groups was diagnosis of renal disease (warfarin $=25.3 \%$ vs $\mathrm{DOAC}=14.0 \%$ ). Other patient characteristics where meaningful differences between groups existed before IPTW included receipt of opioid $($ warfarin $=39.0 \%$ vs DOAC $=26.5 \%)$ and nonopioid (warfarin $=49.0 \%$ vs
DOAC $=27.7 \%)$ analgesics, diuretics (51.6\% vs 39.1\%), and ATRIA score (3.52 vs 3.06).

\section{UNADJUSTED RESULTS}

Survival was lower among warfarin patients, with $73.5 \%$ of patients alive at the end of year 3 compared with $80.8 \%$ of DOAC patients. Mean years of follow-up were 1.48 years (interquartile range $[\mathrm{IQR}]=0.70-2.24$ years) and 1.24 years $(\mathrm{IQR}=0.56-1.74$ years) for patients receiving warfarin and DOACs, respectively. Across all patients, mean (SD) unadjusted total 3-year medical expenditure was $\$ 56,265(\$ 96,666)$ for the warfarin group compared with $\$ 32,736(\$ 52,470)$ for the DOAC group (Table 3). Mean medical expenditures were higher in the warfarin group compared with the DOAC group among patients who were alive at the start of each year of followup. For warfarin patients, medical (SD) expenditures declined from $\$ 39,951$
$(\$ 80,606)$ in year 1 to $\$ 28,196(\$ 58,372)$ in year 3. For DOAC patients, medical (SD) expenditures were similar across years, ranging from $\$ 23,916(\$ 41,026)$ in year 1 to $\$ 24,601(\$ 45,863)$ in year 3. Similar to mean medial expenditure, median estimates were higher for warfarin patients compared with DOAC patients.

Across all patients, mean (SD) unadjusted 3-year anticoagulant expenditure was \$220 (\$537) for the warfarin group compared with $\$ 1,445(\$ 1,270)$ for the DOAC group (Supplementary Table 1, available in online article). Among patients who were alive at the start of each year of follow-up, mean and median anticoagulant expenditures declined in each year but were lower in the warfarin group in all 3 years.

\section{ADJUSTED RESULTS}

AF patients treated with warfarin had 0.52 fewer expected years of survival $(95 \% \mathrm{CI}=-0.695$ to -0.349 ; Supplementary Table 2, available in online article). Warfarin patients also had higher expected annual medical expenditure in years of death $(\$ 9,461$, $95 \% \mathrm{CI}=\$ 5,207$ to $\$ 13,716)$ and survival over the full year $(\$ 10,588,95 \%$ $\mathrm{CI}=\$ 9,392$ to $\$ 11,784)$. Combining estimates for each of these model parts yielded average expected 3-year medical expenditure of $\$ 72,026 \quad(95 \%$ CI $=\$ 70,131$ to $\$ 73,921$; Table 4). Three-year medical expenditure was estimated to be $\$ 25,688$ $(95 \% \mathrm{CI}=\$ 23,122$ to $\$ 28,254)$ greater for warfarin, compared with DOAC patients.

In the secondary analysis, warfarin patients had lower expected anticoagulant expenditure during years of death $(-\$ 346,95 \% \mathrm{CI}=-\$ 393$ to $-\$ 300)$ and survival over the full year $(-\$ 900$, 95\% CI $=-\$ 922$ to $-\$ 878)$. Combining estimates from the multipart model yielded an average expected 3-year 
TABLE 4 Estimated Total Expenditure and Between Group Differences in Expenditure Over 3 Years

\begin{tabular}{|c|c|c|c|c|}
\hline Quantity & Mean & SE & Lower $95 \% \mathrm{Cl}$ & Upper $95 \% \mathrm{Cl}$ \\
\hline \multicolumn{5}{|l|}{ Medical expenditure } \\
\hline Expenditure difference ${ }^{a}$ & 25,688 & 1,309 & 23,122 & 28,254 \\
\hline Total expenditure $^{\mathrm{b}}$ & 72,026 & 967 & 70,131 & 73,921 \\
\hline \multicolumn{5}{|l|}{ Anticoagulant expenditure } \\
\hline Expenditure difference $^{a}$ & $-1,849$ & 31 & $-1,910$ & $-1,788$ \\
\hline Total expenditure ${ }^{\mathrm{b}}$ & 1,162 & 16 & 1,131 & 1,193 \\
\hline \multicolumn{5}{|c|}{$\begin{array}{l}{ }^{a} \text { Expenditure difference represents difference in expected total 3-year ex } \\
\text { patients. } \\
\text { bTotal expenditure represents average expected expenditure across all pc } \\
\text { 'All estimates are inflation adjusted to represent } 2018 \text { constant dollars. } \\
D O A C=\text { direct oral anticoagulant; } S E=\text { standard error. }\end{array}$} \\
\hline
\end{tabular}

expenditure of $\$ 1,162(95 \% \mathrm{CI}=\$ 1,131$ to $\$ 1,193 ;$ Table 4). Three-year anticoagulant drug expenditure was $\$ 1,849$ (95\% $\mathrm{CI}=-\$ 1,910$ to $-\$ 1,788)$ lower for warfarin, compared with DOAC patients.

\section{Discussion}

This study compared downstream expenditures among VA-Medicare dual enrollees who were newly diagnosed with $\mathrm{AF}$ and initiated warfarin or DOAC therapy. Limited evidence exists that examines the downstream economic implications of drug therapies for treatment of AF. Previous research demonstrating the efficacy of DOACs compared with warfarin with respect to clinical outcomes suggests lower downstream costs associated with DOAC treatment. ${ }^{39}$ However, this evidence is primarily from trial settings where the characteristics of patients differ from those in real-world settings.

Findings indicate warfarin patients have statistically significant and substantially greater expected medical expenditure over the 3-year period following treatment initiation, representing approximately $36 \%$ of the mean total expenditure. This difference stemmed from warfarin patients (1) incurring greater expenditure during years when patients survive the full year and (2) incurring greater expenditure during years when death occurs. Greater expenditure in both of these cases is potentially due to health service utilization related to greater rate of stoke and bleeding events among warfarin compared with DOAC patients, as shown in previous research. ${ }^{6,18,21,22}$ Higher medical expenditure among warfarin patients was accompanied by lower anticoagulant drug expenditure. However, the magnitude of this difference was substantially smaller than differences in medical expenditure.

The difference in total 3-year medical expenditure between groups translated to $\$ 714$ per patient per month (PPPM). In comparison, Amin et al found that patients treated with warfarin incurred $\$ 68$ greater in costs related to stroke/SE and \$239 greater in major bleeding-related costs compared with patients treated with apixaban. ${ }^{22}$ Gupta et al examined total health care cost differences between warfarin and 3 DOACs, only finding statistically significant differences between warfarin compared with rivaroxaban (warfarin lower by $\$ 346$ PPPM). ${ }^{23}$

Taken together, our findings demonstrate the downstream economic benefit of DOACs compared with warfarin for incident cases of AF in VA patients aged 65 years and over. These findings coincide with results of previous studies demonstrating the clinical efficacy of DOACs. The approximately $\$ 8,563$ lower expected per year medical expenditure among DOAC compared with warfarin patients exceeds the higher annual costs of anticoagulant prescriptions estimated in this study (\$616). Lower annual medical expenditure estimated in this study also more than offsets higher annual prescription costs between the DOAC dabigatran and generic warfarin (\$1,354 vs $\$ 113)$ found outside of the VA. ${ }^{40,41}$

Study findings present differences on average across the VA population; however, the economic benefit of DOACs may differ within subgroups such as patients with kidney disease where the clinical efficacy is less clear.

Findings from this study also address concerns that the results from trial settings may not translate to real-world settings such as the VA. The economic benefit of DOACs materialized even in a population with known challenges to medication adherence and greater prevalence of comorbid conditions. Our results are one component that stakeholders such as pharmacy benefits management services should consider when maintaining prescription formularies and determining prescription prices. The results are also of relevance for administrators responsible for projecting expected costs of care for a given patient population.

\section{LIMITATIONS}

This study has several limitations. First, by design, our study focused on the population of VA patients, in part 
to determine whether previous findings of DOAC efficacy translate to this population. Findings from this study should be generalized accordingly.

Second, we classified patients initiating 1 of 5 DOACs into a single category due to power considerations. It is possible that the economic benefits of DOACs compared with warfarin in this study may differ across DOAC classes.

Third, we applied machine learning tools to develop a propensity score model using an extensive set of patient covariates to address potential selection bias in treatment choice. Other omitted variables influencing choice of pharmacotherapy may confound study results.

Fourth, nursing home expenditures in the VA capture postacute care and long-term care but only include the former in Medicare due to reimbursement policies. ${ }^{42}$

Finally, we examined dual VA-FFS Medicare enrollees to increase completeness in data capturing medical expenditure. It is possible differences in the costs of care received outside of the VA and Medicare between groups may influence estimates in this study and may include long-term care services paid for by Medicaid.

\section{Conclusions}

Among VA patients over age 65 years with incident $\mathrm{AF}$, total medical expenditure in the 3 years following initial diagnosis was markedly greater for patients treated with warfarin compared with DOACs. This aligns with previous evidence that demonstrates the clinical benefits of DOACs in randomized trials and other non-VA populations. Study findings are of relevance to stakeholders charged with administering pharmacy benefits and measuring the expected costs of care for patients with this increasingly common condition.

\section{DISCLOSURES}

This study was funded by a VA Health Services Research and Development Investigator Initiated Research Award (IIR 15-139). Support for VA/CMS data was provided by the Department of Veterans Affairs, Veterans Health Administration, Office of Research and Development, Health Services Research and Development, VA Information Resource Center (Project Numbers SDR 02-237 and 98-004). The funder had no role in study design, data collection and analysis, decision to publish, or preparation of the manuscript. The views expressed in this article are those of the authors and do not necessarily reflect the position or policy of the Department of Veterans Affairs, the University of Washington, Northeastern University, and Boston University. The authors declare no conflicts of interest.

This research includes data obtained from the VHA Office of Performance Measurement (17API2), which resides within the Office of Analytics and Performance Integration (API), under the Office of Quality and Patient Safety (QPS; formerly known as RAPID).

An oral presentation documenting a subset of the findings from this study was presented at the 2020 AcademyHealth Annual Research Meeting, delivered virtually on July 29, 2020.

\section{REFERENCES}

1. Centers for Disease Control and Prevention. Atrial fibrillation. 2020. Accessed August 20, 2020. https://www. cdc.gov/heartdisease/atrial fibrillation. $\underline{\text { htm }}$

2. Mas Dalmau G, Sant Arderiu E, Enfedaque Montes MB, Sola I, Pequeno Saco S, Alonso Coello P. Patients' and physicians' perceptions and attitudes about oral anticoagulation and atrial fibrillation: a qualitative systematic review. BMC Fam Pract. 2017;18(1):3.

3. Ruff CT, Giugliano RP, Braunwald E, et al. Comparison of the efficacy and safety of new oral anticoagulants with warfarin in patients with atrial fibrillation: a meta-analysis of randomised trials. Lancet. 2014;383(9921):955-62.
4. Pan KL, Singer DE, Ovbiagele B, Wu YL, Ahmed MA, Lee M. Effects of nonvitamin $\mathrm{K}$ antagonist oral anticoagulants versus warfarin in patients with atrial fibrillation and valvular heart disease: a systematic review and meta-analysis. J Am Heart Assoc. 2017;6(7):e005835.

5. Liberato NL, Marchetti M. Costeffectiveness of non-vitamin $\mathrm{K}$ antagonist oral anticoagulants for stroke prevention in non-valvular atrial fibrillation: a systematic and qualitative review. Expert Rev Pharmacoecon Outcomes Res. 2016;16(2):221-35.

6. Vinogradova Y, Coupland C, Hill T, Hippisley-Cox J. Risks and benefits of direct oral anticoagulants versus warfarin in a real world setting: cohort study in primary care. BMJ. 2018;362:k2505.

7. Edens EL, Kasprow W, Tsai J, Rosenheck RA. Association of substance use and VA service-connected disability benefits with risk of homelessness among veterans. Am J Addict. 2011;20(5):412-19.

8. Teeters JB, Lancaster CL, Brown DG, Back SE. Substance use disorders in military veterans: prevalence and treatment challenges. Subst Abuse Rehabil. 2017;8:69-77.

9. Lwi SJ, Barnes DE, Xia F, Peltz C, Hoang T, Yaffe K. Ten-year prevalence of cognitive impairment diagnoses and associated medical and psychiatric conditions in a national cohort of older female veterans. Am J Geriatr Psychiatry. 2019;27(4):417-25.

10. Agha Z, Lofgren RP, Van Ruiswyk JV, Layde PM. Are patients at Veterans Affairs medical centers sicker? A comparative analysis of health status and medical resource use. Arch Intern Med. 2000;160(21):3252-57.

11. Stollberger C, Finsterer J. Concerns regarding the use of dabigatran for stroke prevention in atrial fibrillation. Pharmaceuticals (Basel). 2012;5(2):155-68.

12. Stanton BE, Barasch NS, Tellor KB. Comparison of the safety and effectiveness of apixaban versus warfarin in patients with severe renal impairment. Pharmacotherapy. 2017;37(4):412-19. 
13. Jain N, Reilly RF. Clinical pharmacology of oral anticoagulants in patients with kidney disease. Clin J Am Soc Nephrol. 2019;14(2):278-87.

14. Skeppholm M, Hjemdahl P, Antovic JP, et al. On the monitoring of dabigatran treatment in "real life" patients with atrial fibrillation. Thromb Res. 2014;134(4):783-89.

15. Villines TC, Schnee J, Fraeman K, et al. A comparison of the safety and effectiveness of dabigatran and warfarin in non-valvular atrial fibrillation patients in a large healthcare system. Thromb Haemost. 2015;114(6):1290-98.

16. Graham DJ, Reichman ME, Wernecke M, et al. Cardiovascular, bleeding, and mortality risks in elderly Medicare patients treated with dabigatran or warfarin for nonvalvular atrial fibrillation. Circulation. 2015;131(2):157-64.

17. Lauffenburger JC, Farley JF, Gehi AK, Rhoney DH, Brookhart MA, Fang G. Effectiveness and safety of dabigatran and warfarin in real-world US patients with non-valvular atrial fibrillation: a retrospective cohort study. J Am Heart Assoc. 2015;4(4):e001798.

18. Larsen TB, Skjoth F, Nielsen PB, Kjaeldgaard JN, Lip GY. Comparative effectiveness and safety of non-vitamin $\mathrm{K}$ antagonist oral anticoagulants and warfarin in patients with atrial fibrillation: propensity weighted nationwide cohort study. BMJ. 2016;353:i3189.

19. Li WH, Huang D, Chiang CE, et al. Efficacy and safety of dabigatran, rivaroxaban, and warfarin for stroke prevention in Chinese patients with atrial fibrillation: the Hong Kong Atrial Fibrillation Project. Clin Cardiol. 2017;40(4):222-29.

20. Go AS, Singer DE, Toh S, et al. Outcomes of dabigatran and warfarin for atrial fibrillation in contemporary practice: a retrospective cohort study. Ann Intern Med. 2017;167(12):845-54.

21. Yao X, Abraham NS, Sangaralingham LR, et al. Effectiveness and safety of dabigatran, rivaroxaban, and apixaban versus warfarin in nonvalvular atrial fibrillation. J Am Heart Assoc. 2016;5(6):e003725.
22. Amin A, Keshishian A, Trocio J, et al. Risk of stroke/systemic embolism, major bleeding and associated costs in nonvalvular atrial fibrillation patients who initiated apixaban, dabigatran or rivaroxaban compared with warfarin in the United States Medicare population. Curr Med Res Opin. 2017;33(9):1595-604.

23. Gupta K, Trocio J, Keshishian A, et al. Real-world comparative effectiveness, safety, and health care costs of oral anticoagulants in nonvalvular atrial fibrillation patients in the U.S. Department of Defense population. J Manag Care Spec Pharm. 2018;24(11):1116-27. doi: 10.18553/ jmcp.2018.17488

24. Barnett PG. Determination of VA health care costs. Med Care Res Rev. 2003;60(3 Suppl):124S-141S.

25. VA Health Economics Resource Center. Managerial Cost Accounting (MCA). 2021. Accessed Feb 1, 2021. https://www. herc.research.va.gov/include/page. asp?id=managerial-cost-accounting

26. Goulet JL, Erdos J, Kancir S, et al. Measuring performance directly using the Veterans Health Administration electronic medical record: a comparison with external peer review. Med Care. 2007;45(1):73-79.

27. Kubota K, Ooba N, Kamijima Y, Sato K, Koide D. The use of anticoagulants in patients with non-valvular atrial fibrillation between 2005 and 2014: a drug utilization study using claims data in Japan. PLoS One. 2018;13(9):e0203380.

28. Bureau of Labor Statistics. Consumer Price Index. 2021. Accessed Feb 1, 2021. https://www.bls.gov/cpi/

29. Berndt ER, Cutler DM, Frank RG, Griliches Z, Newhouse JP, Triplett JE. Price indexes for medical care goods and services-an overview of measurement issues. In: Cutler DM, Berndt ER, eds. Medical Care Output and Productivity. University of Chicago Press; 2001.
30. Lip GY, Nieuwlaat R, Pisters R, Lane DA, Crijns HJ. Refining clinical risk stratification for predicting stroke and thromboembolism in atrial fibrillation using a novel risk factor-based approach: the euro heart survey on atrial fibrillation. Chest. 2010;137(2):263-72.

31. Fang MC, Go AS, Chang Y, et al. A new risk scheme to predict warfarinassociated hemorrhage: the ATRIA (Anticoagulation and Risk Factors in Atrial Fibrillation) Study. J Am Coll Cardiol. 2011;58(4):395-401.

32. Senoo K, Proietti M, Lane DA, Lip GY. Evaluation of the HAS-BLED, ATRIA, and ORBIT bleeding risk scores in patients with atrial fibrillation taking warfarin. Am J Med. 2016;129(6):600-07.

33. Turagam MK, Velagapudi P, Flaker GC. Stroke prevention in the elderly atrial fibrillation patient with comorbid conditions: focus on non-vitamin $\mathrm{K}$ antagonist oral anticoagulants. Clin Interv Aging. 2015;10:1431-44.

34. Poli D, Antonucci E, Pengo V, Testa S, Palareti G. Comparison of HAS-BLED and HAS-BED versus CHADS2 and CHA2DS2VASC stroke and bleeding scores in patients with atrial fibrillation. Am J Cardiol. 2017;119(7):1012-16.

35. Casciano JP, Dotiwala ZJ, Martin BC, Kwong WJ. The costs of warfarin underuse and nonadherence in patients with atrial fibrillation: a commercial insurer perspective. J Manag Care Pharm. 2013;19(4):302-16. doi: 10.18553/ jmcp.2013.19.4.302

36. McCaffrey DF, Ridgeway G, Morral AR. Propensity score estimation with boosted regression for evaluating causal effects in observational studies. Psychol Methods. 2004;9(4):403-25.

37. Basu A, Manning WG. Estimating lifetime or episode-of-illness costs under censoring. Health Econ. 2010;19(9):1010-28.

38. Austin PC. Balance diagnostics for comparing the distribution of baseline covariates between treatment groups in propensity-score matched samples. Stat Med. 2009;28(25):3083-107. 
39. Wadhera RK, Russell CE, Piazza G. Cardiology patient page. Warfarin versus novel oral anticoagulants: how to choose? Circulation. 2014;130(22):e191-93.

40. VA Office of Procurement Acquisition and Logistics. Pharmaceutical prices.

2020. Accessed August 20, 2020. https:// www.va.gov/opal/nac/fss/pharmPrices. $\underline{\text { asp }}$
41. Cowper PA, Sheng S, Lopes RD, et al. Economic analysis of apixaban therapy for patients with atrial fibrillation from a US perspective: results from the ARISTOTLE randomized clinical trial. JAMA Cardiol. 2017;2(5):525-34.
42. Centers for Medicare \& Medicaid Services. Medicare Part A coverage nursing home care. 2020. Accessed August 20, 2020. https://www.medicare.gov/what-medicare-covers/ what-part-a-covers/medicare-part-acoverage-nursing-home-care 